

\title{
Linear Solvation Energy Relationship of Some Aromatic Compounds in RP-HPLC
}

\author{
Y. JIN ${ }^{1,4}$, L.H. YAN ${ }^{2}$, M.J. JIN ${ }^{3}$, Y.D. CHENG ${ }^{1}$ and K.H. Row ${ }^{4, *}$
}

${ }^{1}$ College of Food Science and Technology, Shanghai Ocean University, Shanghai, 201-306, P.R. China

${ }^{2}$ Department of Mathematics, Inha University, Incheon, 402-751, South Korea

${ }^{3}$ Department of Forestry Science, Agricultural College of Yanbian University, Yanji, Jilin, 133-002, P.R. China

${ }^{4}$ Department of Chemical Engineering, Inha University, Incheon, 402-751, South Korea

*Corresponding author: Fax: +82 32 8720959; Tel: +82 32 8607470; E-mail: rowkho@inha.ac.kr

(Received: 6 March 2012;

Accepted: 7 January 2013)

AJC-12662

\begin{abstract}
The fundamental chemical interactions governing the retention of 5 aromatic compounds such as benzene, toluene, chlorobenzene, $o$-xylene and 1,2-dichlorobenzene were investigated in methanol/water mobile phase on a $\mathrm{C}_{18}$ column using linear solvation energy relationship (LSER) model. The ability of the linear solvation energy relationship to account for the chemical interactions underlying solute retention was shown. A comparison of predicted and experimental retention factors suggests that linear solvation energy relationship formalism is able to reproduce adequately the experimental retention factors of the solutes studied in the different experimental conditions investigated. The predicted retention factors by the linear solvation energy relationship model have good agreement with experimental data in the employed mobile phase conditions.
\end{abstract}

Key Words: Linear solvation energy relationship, Aromatic compounds, Retention factor, Reverse phase, High performance liquid chromatography.

\section{INTRODUCTION}

A widely used parameter in reversed-phase high-performance liquid chromatography (RP-HPLC) is the retention factor $(\mathrm{k})$ of a compound in isocratic elution ${ }^{1}$. Many chemical substances are actually complex mixtures of organic chemicals and environmental contamination is almost invariably caused by mixtures of compounds. Recently, several experimental methods were developed for which a fractionation according to hydrophobicity is required for complex mixtures, which contain organic micropollutants of diverse structures. This fractionation can be used to perform tests for the presence of potentially bioconcentrating compounds in environmental samples or to obtain information on the hydrophobicity distribution profile of complex mixtures of unknown composition ${ }^{1}$.

Retention prediction and selectivity optimization are very important in rapid method development in reversed-phase liquid chromatography (RPLC). However, retention in RPLC is a complicated process that depends on many physical and chemical properties of the system, such as temperature, solute molecular properties, stationary phase characteristics and mobile phase composition. These years many practical retention models for RP-HPLC, such as linear solvation energy relationships (LSER), have been developed and widely used ${ }^{2}$.
Quantitative structure-activity relationship (QSAR) theory was developed in the form of a linear solvation energy relationship (LSER) by Kamlet et al. ${ }^{3}$, who used a multi-parameter approach to describe configurational properties in terms of a solute cavity in a solvent and the solvent-solute interactions. Since then, the theory has been adapted to other aspects of chemistry including reaction rates, toxicity, fluorescence lifetimes and others. Linear solvation energy relationship methods involve the application of solvent parameters in linear or multiple-linear regression formulations to express solvent effects for property and reactivity prediction. Properties of mixtures, such as chromatographic retentions, water-octanol partition coefficients and solubilities have been successfully predicted using linear solvation energy relationship methods $\mathrm{s}^{4,5}$.

Recently, many practical retention models ${ }^{1,6,7}$ for RPHPLC, such as linear solvation energy relationships (LSER), have been developed and widely used. In this study, we have used linear solvation energy relationship to explain retention in RP-HPLC using methanol and water mobile phase.

The general linear solvation energy relationship equation used in this work is ${ }^{8}$ :

$$
\log \mathrm{k}=\log \mathrm{k}_{0}+\mathrm{m}\left(\frac{\mathrm{V}_{\mathrm{x}}}{100}\right)+\mathrm{s} \pi_{2}^{\mathrm{H}}+\mathrm{a} \Sigma \alpha_{2}^{\mathrm{H}}+\mathrm{b} \Sigma \beta_{2}^{\mathrm{H}}+\mathrm{rR}_{2}
$$


where, $\mathrm{k}$ is the experimental retention factor. The $\mathrm{V}_{\mathrm{x}}, \pi_{2}{ }^{\mathrm{H}}, \Sigma \alpha_{2}{ }^{\mathrm{H}}$, $\Sigma \beta_{2}{ }^{\mathrm{H}}$ and $\mathrm{R}_{2}$ terms are solute descriptors, where $\mathrm{V}_{\mathrm{x}}$ represents the solute's size/polarizability, $\pi_{2}{ }^{\mathrm{H}}$ is the dipolarity/polarizability, $\Sigma \alpha_{2}{ }^{\mathrm{H}}$ is the hydrogen bond (HB) acidity, $\Sigma \beta_{2}{ }^{\mathrm{H}}$ is the HB basicity and $R_{2}$ is the excess molar refraction. The subscript "2" simply signifies that these parameters are solute descriptors.

The coefficients of these descriptors $\mathrm{m}, \mathrm{s}, \mathrm{a}, \mathrm{b}$ and $\mathrm{r}$ reflect differences in the two bulk phases between which the solute is transferring ${ }^{9}$ and are obtained through a multiparameter linear regression. The $\log \mathrm{k}_{0}$ term is simply the intercept of the regression and is comprised of constant contributions from the solutes and the chromatographic system.

We note that since the parameters $\mathrm{V}_{\mathrm{x}}$ and $\pi_{2}{ }^{\mathrm{H}}$ are blends of two different interactions, the coefficients of these parameters are also blends of the corresponding properties. Specifically, $\mathrm{m}$ is the difference in the cohesivity/dispersive ability of the two bulk phases and $\mathrm{s}$ is the difference in the ability of the two phases to interact through dipole-dipole and dipole-induced dipole interactions. Many reviews and examples of linear solvation energy relationships and their interpretations are available ${ }^{10-14}$.

In this study, five aromatic compounds (benzene, toluene, chlorobenzene, $o$-xylene and 1,2-dichlorobenzene) have been in terms of linear solvation energy relationship.

\section{EXPERIMENTAL}

Analytical grade solvents, five standard aromatic compounds, benzene, toluene, chlorobenzene, $o$-xylene and 1,2dichlorobenzene were purchased from Ducksan Pure Chemical (Kyungki-Do, Korea). HPLC grade solvent, methanol was from Ducksan Pure Chemical (Kyungki-Do, Korea). Twice distilled water was filtered by decompressing pump (Division of Millipore, Waters, USA) and filter (FH-0.45 mm).

Sample preparation: Five $0.025 \mathrm{mg}$, standard aromatic compounds were dissolved in $1 \mathrm{~mL}$ of methanol and the concentration of the solutions was adjusted to $25 \mathrm{mg} / \mathrm{mL}$. The constant $10 \mathrm{~mL}$ injection volume of mixtures solution was used throughout.

The HPLC experiments were performed with Shimadzu LC-6AD pump and SIL-10 Avp autosampler (Younglin, Korea) and SPD-M10 Avp PDA detector (Younglin, Korea) and CTS 30 column oven (Younglin, Korea). Sufficient times were allowed for the stabilization of the column and detector signal after each injection and the solvents in the reservoirs were continuously stripped with helium to degas the mobile phase. The flow rate of the mobile phase was $1 \mathrm{~mL} / \mathrm{min}$ and was monitored at the fixed wavelength of $270 \mathrm{~nm}$. The column was purchased from RS-tech Co (Daejeon, Korea). The Waters symmetry column size was $0.46 \mathrm{~cm} \times 15 \mathrm{~cm}$ and packed by $\mathrm{C}_{18}, 100 \AA$, $5 \mu \mathrm{m}$. All the experimental runs were carried out at $30^{\circ} \mathrm{C}$.

Retention factor estimation: The retention factor, $\mathrm{k}$, of each solute was measured according to the following formula:

$$
\mathrm{k}=\frac{\left(\mathrm{t}_{\mathrm{R}}-\mathrm{t}_{\mathrm{M}}\right)}{\mathrm{t}_{\mathrm{M}}}
$$

here, $t_{R}$ and $t_{M}$ are the retention times of the retained analyte and the retention times of the unretained analyte (also known as dead time), respectively. To calculate a retention factor, the hold-up time of the used HPLC system should be measured. From the breakthrough curves without column installing, the mixer volume of system was measured as $4.385 \mathrm{~mL}$ and the dead time of the column was measured as $1.434 \mathrm{~min}$ with $\mathrm{KNO}_{3}$ injection. Three replicate injections were made to determine the retention time of each substance and the average values of at least three determinations were used to calculate the retention factors. Evaluation of the results of the chromatographic experiments was carried out using mathematical statistic techniques.

Linear solvation energy relationship estimations: Retention factors were determined for the five aromatic compounds used in this study and the system constants were calculated by multiple linear regressions using Origin Pro 6.0 software (Microcal Software Inc., MA, USA). The differences in LSER coefficients indicate the variations in the types of interactions between stationary phases and solutes. In the column, mechanisms such as solute interacting with mobile phase and coating to stationary phase occur during the separated process. Due to these different mechanisms, the LSER constants for different kinds of solutes are not identical.

\section{RESULTS AND DISCUSSION}

The retention behaviours of the five aromatic compounds (benzene, toluene, chlorobenzene, $o$-xylene and 1,2-dichlorobenzene) in each mobile phase were examined and compared using the solvation parameter LSER model, i.e., model described in eqn. 2 . The test solutes and their descriptors used in this study are given in Table-1.

\begin{tabular}{lcccccc}
\multicolumn{7}{c}{ TABLE-1 } \\
TEST SOLUTES AND THEIR DESCRIPTORS \\
FOR THE SOLVATION PARAMETER MODEL \\
\cline { 2 - 7 } \multicolumn{1}{c}{ Solute } & $\begin{array}{c}\mathrm{V}_{\mathrm{X}} \\
\left(\mathrm{cm}^{3} / \mathrm{mol}^{-1}\right)\end{array}$ & $\pi_{2}{ }^{\mathrm{H}}$ & $\alpha_{2}{ }^{\mathrm{H}}$ & $\beta_{2}{ }^{\mathrm{H}}$ & $\begin{array}{c}\mathrm{R}_{2} \\
\left(\mathrm{~cm}^{3} / 10\right)\end{array}$ \\
\hline Benzene & 0.7160 & 0.52 & 0 & 0.14 & 0.6100 \\
Toluene & 0.8573 & 0.52 & 0 & 0.14 & 0.6010 \\
Chlorobenzene & 0.8388 & 0.65 & 0 & 0.07 & 0.7180 \\
o-Xylene & 0.9982 & 0.56 & 0 & 0.16 & 0.6630 \\
1,2-Dichlorobenzene & 0.9612 & 0.78 & 0 & 0.04 & 0.8720 \\
\hline
\end{tabular}

The coefficients for the linear solvation energy relationship equations obtained for methanol aqueous mobile phases were listed in Table-2 and Fig. 1. In all the mobile phase investigated most of the values of $b$ and $s$ were negative, it mean that an increase in the hydrogen bond basicity and solute dipolarity/polarizability decreases the overall retention of the molecule. Furthermore, most of the values of $m$ were positive in all studies, indicating that increases in the solute volume, excess molar and hydrogen bond acidity will make increases in the solute volume and excess molar. In view of value range of coefficients, the excess molar refractivity $\left(\mathrm{R}_{2}\right)$ and solute dipolarity/polarizability $\left(\mathrm{V}_{\mathrm{x}}\right)$ generally play the largest role in determining the retention of solutes in all studies. Hydrogen bond basicity $\left(\Sigma \beta_{2}{ }^{\mathrm{H}}\right)$ is also an important factor in the each mobile phase with coefficients comparable in magnitude to those of solute volume. 
TABLE-2

CONSTANTS FOR THE LIQUID CHROMATOGRAPHY SYSTEMS USING SOLVATION PARAMETER MODEL

\begin{tabular}{cccccccc}
\hline & \multicolumn{6}{c}{ Methanol compositions (\%) } \\
\cline { 2 - 8 } & & 36 & 44 & 52 & 60 & 68 & 76 \\
\hline \multirow{5}{*}{ Constants } & $\mathrm{r}$ & 2.36 & 1.24 & 2.51 & 0.70 & 0.89 & 1.73 \\
& $\mathrm{~b}$ & -4.17 & -2.97 & -3.78 & -1.61 & -1.66 & -2.09 \\
& $\mathrm{a}$ & - & - & - & - & - & - \\
& $\mathrm{s}$ & -3.56 & -2.08 & -3.90 & -1.24 & -1.56 & -2.68 \\
& $\mathrm{~m}$ & 2.75 & 2.38 & 2.17 & 1.74 & 1.52 & 1.32 \\
& $\mathrm{c}$ & -0.29 & -0.47 & -0.23 & -0.71 & -0.70 & -0.61 \\
\hline \multirow{2}{*}{ Statistics } & $\mathrm{r}^{2}$ & 0.9999 & 0.9994 & 0.9971 & 0.9909 & 0.9838 & 0.9718 \\
& $\mathrm{SD}$ & 0.3052 & 0.2619 & 0.2214 & 0.1854 & 0.1548 & 0.1262 \\
\hline
\end{tabular}

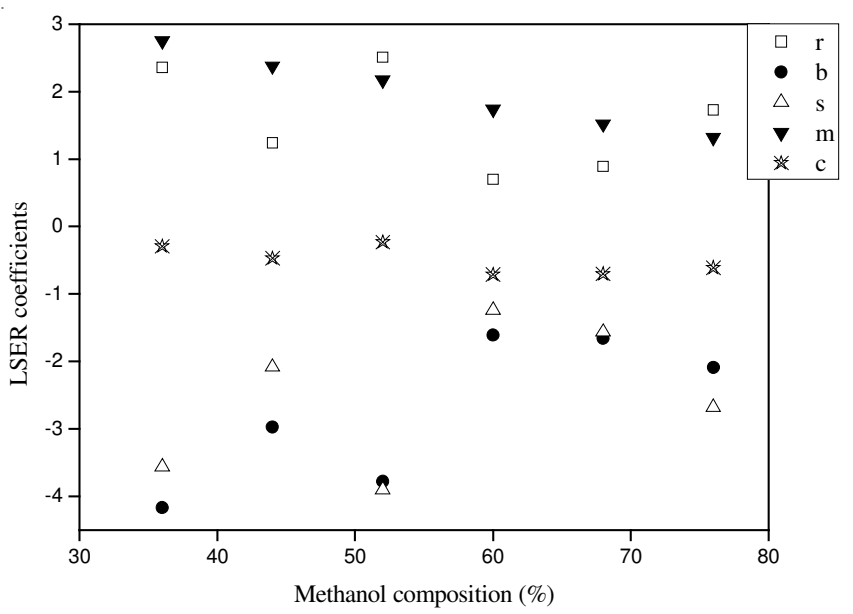

Fig. 1. Linear solvation energy relationship coefficients as a function of methanol mobile phase compositions

As shown in Fig. 1, the values of all of the 5 coefficients ( $\mathrm{m}, \mathrm{s}, \mathrm{c}, \mathrm{b}$ and $\mathrm{r}$ ) change very complicatedly as the concentrations of methanol changing. In these RP-HPLC conditions, the value of $m$ was positive. According to eqn. 1, a positive sign of $\mathrm{m}$ indicates that the solute will preferentially transfer from the aqueous phase to the surfactant phase. As the concentration of the methanol changing the value of $m$ changed very complex, it can reflect the effect of the methanol content was very important for the retention of 5 solvents from side.

The difference in dipolarity/polarizability is represented by the coefficient s. A negative sign for this coefficient indicates that the solutes experience a microenvironment that has less dipolar/polarizable characteristics than the aqueous mobile phases. All of the values of $\mathrm{s}$ were negative in methanol aqueous mobile phase system. As the concentration of methanol increasing, the trend of $\mathrm{s}$ was increased to a maximum -1.24 and then decreased.

The coefficient ' $a$ ' is an important factor in the solvatochromic model in the two systems studied here. This coefficient represents the difference in the hydrogen bond accepting basicity of the ionic liquid mobile phase and that of the aqueous phase. All of the values of a of methanol aqueous mobile phase system were can not calculated. Because all of the hydrogen bond (HB) acidities of these five aromatic compounds were zero.

The coefficient ' $b$ ' is the second most important factor in the linear solvation energy relationship solvation parameter model used in this study. A comparison of the coefficients for each concentration of methanol reveals that $b$ and $r$ have the largest absolute values among all coefficients for all concentrations presented here. The $\mathrm{b}$ coefficient is proportional to the difference in the hydrogen bond donating ability of the mobile phase of the methanol aqueous phase. A larger ' $b$ ' coefficient reflects that more solutes partition out of the aqueous environment of the mobile phase and the average solute environment appears to be less polar and higher hydrogen bond donating ability strength of the mobile phase.

As discussed in an earlier study, the ' $r$ ' coefficient represents the excess molar refraction of the solute. All methanol mobile phases have a positive coefficient ' $r$ '. With the component of methanol in mobile phases and the concentrations of methanol increasing, the coefficient $r$ decreased to a minimum 0.70 and then increased. It shows that the stationary phase is slightly better able to bond with polar molecules than in the mobile phase and it is statistically significant for all methanol mobile phase conditions.

In view of the analysis above for 5 coefficients and using previous study 1 for reference, we got the relationship between the concentration of methanol and the chromatographic retention that, the significant a mobile phase leads to competition between methanol and solutes absorbing to the silica, resulting in decreases in the retention times, but with a further increase in the concentration of methanol, hydroxyl groups interact with the silanols groups through electrostatic interactions, producing a weak bilayer electronic structure, which repulses basic sorbates and interacts with the alkyl groups through hydrophobic and non-specific interactions, so the retention of solutes decreases under due to these repulsive and hydrophobic interactions ${ }^{14}$.

Calculated $\log \mathrm{k}$ values of the five aromatic compounds were computed for methanol aqueous mobile phases using eqn. 2. And the relationship between calculated (cal) and experimental (exp) $\log \mathrm{k}$ were showed in Fig. 2. The solvation parameter model is found to provide statistically and chemically results. The correlation between experimental (exp) and calculated (cal) $\log \mathrm{k}$ (mobile phases composed from methanol $36-76 \%$ (v/v) demonstrated in Fig. 2. It showed that LSERs are able to approximately reproduce the experimental $\log \mathrm{k}$ values for the solutes studied in the different methanol aqueous mobile phases.

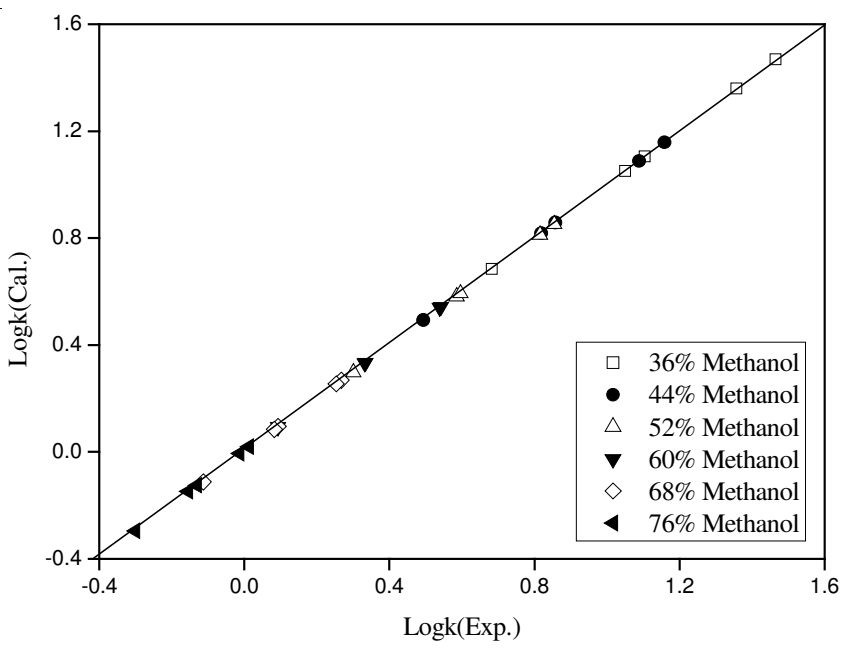

Fig. 2. Correlation between experimental (exp) and calculated (cal) $\log \mathrm{k}$ 


\section{Conclusion}

Five aromatic compounds applied as solutes in the mobile phases using methanol as modifier. The LSER model, i.e., the solvation parameter model, was successfully applied to investigate the effect of the additive concentrations on retention of five aromatic compounds in RP-HPLC. The results obtained from the solvation parameter model provide comparable information, for example, coefficient $s$ and coefficient $r$ play the most important role in retention behavior in methanol mobile phase conditions. It is worth noting that, using the obtained LSER models, it is possible to predict retention factors with high correlation coefficients $\left(r^{2}>0.97\right)$. It is evident from the results of the LSER model that the excess molar refraction and HB basicity have dominant effects role on the solute hydroxyl group interaction. This model is a helpful tool to understand the solute-organic compound interactions and evaluate the retention characteristic of liquid chromatography.

\section{ACKNOWLEDGEMENTS}

This research was supported by the Leading Academic Discipline Project of Shanghai Municipal Education CommissionFood Economic Management (J50703) and the Shanghai Youqing Program Grant (SSC09009).

\section{REFERENCES}

1. Y. Jin, J.W. Lee, C.H. Jin and K.H. Row, Sep. Sci. Technol., 43, 331 (2008).

2. Y. Wang, D. Han, M. Tian and K.H. Row, Korean J. Chem. Eng., 26, $812(2009)$

3. M.J. Kamlet, R.M. Doherty, M.H. Abraham, Y. Marcus and R.W. Taft, J. Phys. Chem., 92, 5244 (1988).

4. M.J. Kamlet, R.M. Doherty, P.W. Carr, D. Mackay, M.H. Abraham and R.W. Taft, Environ. Sci. Technol., 22, 503 (1988).

5. T. Wang, X. Wang and R.L. Smith Jr., J. Supercrit. Fluids, 35, 18 (2005).

6. Y. Jin and K.H. Row, J. Liq. Chromatogr. Rel. Technol., 30, 335 (2007).

7. C.H. Lochmüller, C. Reese, A.J. Aschman and S.J. Breiner, J. Chromatogr. A, 656, 3 (1993).

8. M.H. Abraham, J. Andonian-Haftvan, G.S. Whiting, A. Leo and R.S. Taft, J. Chem. Soc., 8, 1777 (1994).

9. B.W. Gung, X.W. Xue and W.R. Roush, J. Am. Chem. Soc., 125, 3668 (2003).

10. P.W. Carr, R.M. Doherty, M.J. Kamlet, R.W. Taft, W. Melander and C. Horvath, Anal. Chem., 58, 2674 (1986).

11. L.C. Tan and P.W. Carr, J. Chromatogr. A, 799, 1 (1998).

12. M.H. Abraham, H.S. Chadha and A.J. Leo, J. Chromatogr. A, 685, 203 (1994).

13. J.H. Park, J.J. Chae, T.H. Nah and M.D. Jang, J. Chromatogr. A, 664, 149 (1994).

14. M. Tian, S. Li and K.H. Row, Korean J. Chem. Eng., 28, 357 (2011). 